\section{PS10:189 NEUROPSYCHIATRIC LUPUS. A SEVERE MANIFESTATION OF SYSTEMIC LUPUS ERYTHEMATOSUS}

P Athanassiou, C Katsavouni, M Gatsiou, A Tzanavari, C Gerodimos. Department of Rheumatology, St. Paul's Hospital, Thessaloniki, Greece

\subsection{6/lupus-2018-abstract.228}

Purpose Nervous system involvement in systemic lupus erythematosus (SLE) is a grave manifestation of the disease affecting health, quality of life and disease outcome. It is one of the most complex manifestations of SLE and may affect the central, peripheral and autonomous nervous system. Complex interrelated pathogenetic mechanisms are involved in disease pathogenesis. The aim of the study was to describe a patient with neuropsychiatric lupus.

Methods A patient, female aged 50 years presented with SLE with a duration of 20 years. The diagnosis of the disease was made when she presented with intense fatigue, hair loss, a light sensitive rash, arthralgias and positive antinuclear and anti-dsDNA antibodies. In the course of the disease the patient developed CNS involvement with epileptic convulsions, permanent dysarthria and delusions. A brain MRI scan was without specific alterations, however an EEG performed was abnormal and a brain single-photon emission CT (SPECT) revealed decreased perfusion of both frontal and parietal lobes. The patient developed musculoskeletal manifestations at many stages of the disease. At diagnosis the patient had arthralgias of both wrists and knees. The patient presented with a flare with fatigue, mouth ulcers, convulsions, decreased ability to concentrate, intense delusions and dysarthria. At disease flare, when neuropsychiatric symptoms evolved she had diffuse arthralgias.

Results Pulse methylprednisolone i.v. followed by pulse cyclophosphamide i.v. were administered in order to achieve remission. Disease stabilisation was induced by pulse cyclophosphamide at bimonthly intervals and orally administered prednisolone. When remission of the disease was induced by pulse methylprednisolone and cyclophosphamide the patient developed muscle weakness. At disease stabilisation with pulse cyclophosphamide at bimonthly intervals the patient developed arthritis of the hand joints. The disease is now in remission, corticosteroid doses having been significantly reduced.

Conclusions Neuropsychiatric lupus is a grave and complex manifestation of SLE. The disease may be accompanied by various manifestations and severely affects quality of life. Neuropsychiatric lupus should be aggressively treated in order to improve quality of life and disease outcome.

\section{PS10:190 UNUSUAL PRESENTATION OF LUPUS PROFUNDUS}

PD Rath, A Abrari, S Budhiraja. Max Super Speciality Hospital, New Delhi, India

10.1136/lupus-2018-abstract.229

A middle aged female presented with swelling of face and periorbital region for 4 weeks and fever 3 weeks diagnosed and treated as cellulitis elsewhere examination revealed periorbital puffiness and indurated lesions on face with complete distortion of facial features.
Labs-hb- $10.7 \mathrm{gm} / \mathrm{dl}$

Tlc-4500/cumm

Plt- $160 \times 10^{\wedge} 3 /$ microlitre

Sr creatinine- $0.8 \mathrm{mg} / \mathrm{dl}$

Lft- sgot $-87 \mathrm{u} / \mathrm{L}$, sgpt-64 u/l

Ana if- neg

Ena profile- neg

Apla-neg

La-neg

C3, C4 n

$\mathrm{Ct}$ neck with contrast-diffuse enlargement of $\mathrm{rt}$ parotid gland
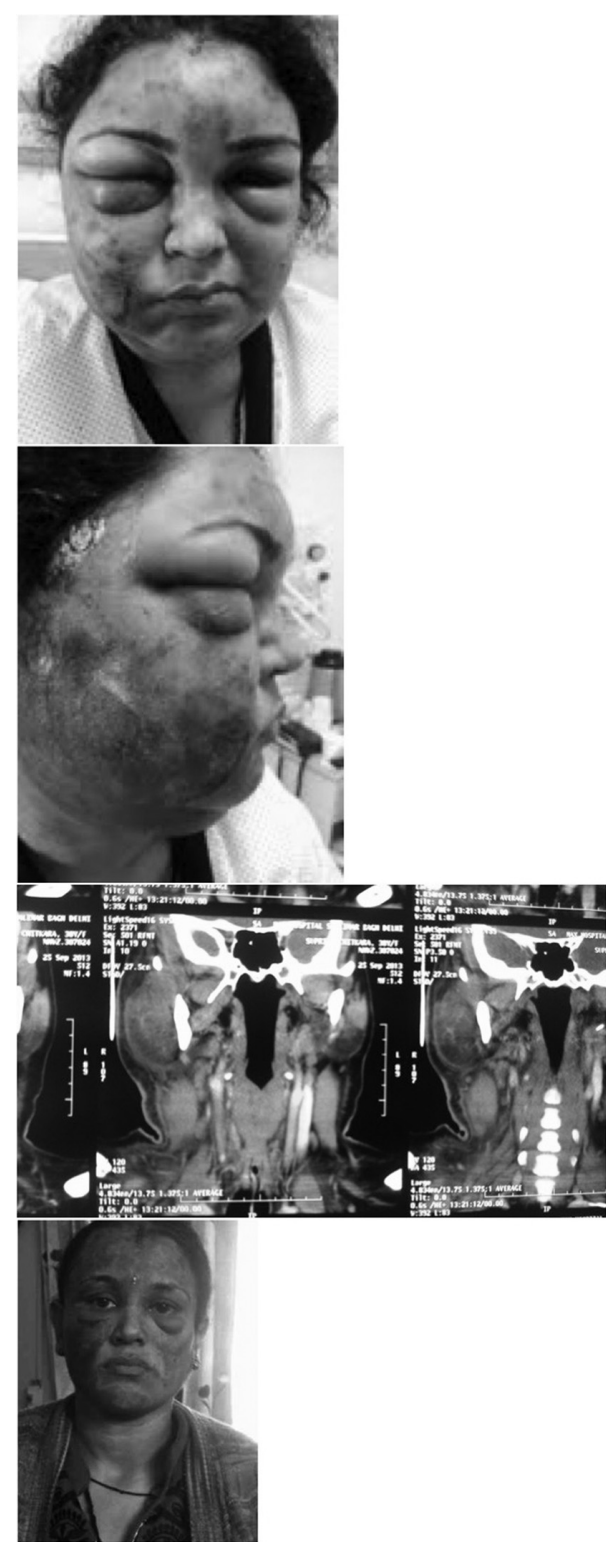

\section{Abstract PS10:190 Figure 1}

Swelling of $\mathrm{rt}$ masseter, buccinator sterno cleido mastoid

Gross oedema of overlying soft tissue of $\mathrm{rt}$ face multiple mildly enlarged $\ln$ rt sub mandibular upper and middle jugular erosion of alveolar cortex of rt post edge of maxilla.

Biopsy was done which showed -the subcut. Panniculus shows a lobular inflammatory infiltrate composed mainly of 